\title{
The composite starburst/AGN nature of the superwind galaxy NGC 4666
}

\author{
M. Persic ${ }^{1}$, M. Cappi ${ }^{2}$, Y. Rephaeli ${ }^{3,4}$, L. Bassani ${ }^{2}$, R. Della Ceca ${ }^{5}$, A. Franceschini ${ }^{6}$, L. Hunt ${ }^{7}$, \\ G. Malaguti ${ }^{2}$, and E. Palazzi ${ }^{2}$
}

1 INAF - Osservatorio Astronomico di Trieste, via GB Tiepolo 11, 34131 Trieste, Italy e-mail: persic@ts.astro.it

2 IASF/CNR - Sezione di Bologna, via P. Gobetti 101, 40129 Bologna, Italy

3 School of Physics and Astronomy, Tel Aviv University, Tel Aviv 69978, Israel

4 CASS, University of California, San Diego, La Jolla, CA 92093, USA

5 INAF/Osservatorio Astronomico di Brera, via Brera 28, 20121 Milano, Italy

${ }^{6}$ Dipartimento di Astronomia, Università di Padova, vicolo Osservatorio 2, 35122 Padova, Italy

7 IASF/CNR - Sezione di Firenze, l.go E. Fermi 5, 50125 Firenze, Italy

Received 25 April 2003 / Accepted 15 June 2004

\begin{abstract}
We report the discovery of a Compton-thick AGN and of intense star-formation activity in the nucleus and disk, respectively, of the nearly edge-on superwind galaxy NGC 4666. Spatially unresolved emission is detected by BeppoSAX only at energies $<10 \mathrm{keV}$, whereas spatially resolved emission from the whole disk is detected by XMM-Newton. A prominent ( $E W \sim 1-2 \mathrm{keV}$ ) emission line at $\sim 6.4 \mathrm{keV}$ is detected by both instruments. From the XMM-Newton data alone the line is spectrally localized at $E \simeq 6.42 \pm 0.03 \mathrm{keV}$, and seems to be spatially concentrated in the nuclear region of NGC 4666 . This, together with the presence of a flat $(\Gamma \sim 1.3)$ continuum in the nuclear region, suggests the existence of a strongly absorbed (i.e., Compton-thick) AGN, whose intrinsic $2-10 \mathrm{keV}$ luminosity is estimated to be $L_{2-10} \gtrsim 2 \times 10^{41} \mathrm{erg} \mathrm{s}^{-1}$. At energies $\lesssim 1 \mathrm{keV}$ the integrated (BeppoSAX) spectrum is dominated by a $\sim 0.25 \mathrm{keV}$ thermal gas component distributed throughout the disk (resolved by $X M M$-Newton). At energies $\sim 2-10 \mathrm{keV}$, the integrated spectrum is dominated by a steep $(\Gamma \gtrsim 2)$ power-law (PL) component. The latter emission is likely due to unresolved sources with luminosity $L \sim 10^{38}-10^{39} \mathrm{erg} \mathrm{s}^{-1}$ that are most likely accreting binaries (with $\mathrm{BH}$ masses $\leq 8 M_{\odot}$ ). Such binaries, which are known to dominate the X-ray point-source luminosity in nearby star-forming galaxies, have $\Gamma \sim 2$ PL spectra in the relevant energy range. A $\Gamma \sim 1.8$ PL contribution from Compton scattering of (the radio-emitting) relativistic electrons by the ambient FIR photons may add a truly diffuse component to the $2-10 \mathrm{keV}$ emission.
\end{abstract}

Key words. X-rays: galaxies - galaxies: starburst - galaxies: Seyfert - galaxies: individual: NGC 4666

\section{Introduction}

The Compton-thick subclass of Seyfert 2 (Sy2) galaxies consists of objects for which the column density of absorbing matter along the l.o.s. to the circumnuclear torus is $N_{\mathrm{H}}>\sigma_{T}^{-1} \simeq$ $1.5 \times 10^{24} \mathrm{~cm}^{-2}$. A column of a few times this critical value is sufficient to strongly depress the transmitted intensity also in hard X-rays, since after a few scatterings the radiation is redshifted into the photoelectrically-dominated regime.

However, even for very high values of $N_{\mathrm{H}}$, nuclear radiation can be observed by the radiation scattered from $(a)$ the visible part of the inner surface of the circum-nuclear torus (that blocks direct X-ray emission entirely from our view; Antonucci 1993), and from scattering by $(b)$ the warm medium that scatters and polarizes the optical broad lines (Antonucci \& Miller 1985). The two scattered components are spectrally different.

(a) The torus, which is presumed to be essentialy neutral, gives rise to the "Compton reflection continuum" or "cold reflection" (see George \& Fabian 1991) that can be explained as follows. Suppose an input power-law (PL) continuum irradiates some "cold" (i.e., $T \lesssim 10^{6}{ }^{\circ} \mathrm{K}$ ) gas with solar chemical abundance. The reflected X-ray spectrum contains the $\mathrm{Fe}-\mathrm{K} \alpha(6.40 \mathrm{keV}), \mathrm{Fe}-\mathrm{K} \beta(7.06 \mathrm{keV})$, and Ni$\mathrm{K} \alpha(7.48 \mathrm{keV})$ fluorescence lines, along with the $\mathrm{Fe}-\mathrm{K}$ shell absorption edge at $\sim 7.11 \mathrm{keV}$. Due to the high abundance and fluorescence yield of iron, a significant fraction of the incident radiation at energies $E \geq 7.11 \mathrm{keV}$ yields the $\mathrm{Fe}-\mathrm{K} \alpha$ fluorescent line at $E=6.40 \mathrm{keV}$. Compton downscattering within the gas causes a fall-off in the continuum at high energies, a smearing of the Fe-K-shell absorption edge, and the formation of low-energy wings of the fluorescence lines. The competition within the slab between multiple electron down-scattering of high-energy photons and photoelectric absorption of low-energy photons leads to a "Compton reflection hump" in the reflected X-ray continuum in the $\sim 20-100 \mathrm{keV}$ band. 
(b) The warm mirror, which is presumed to be highly ionized (i.e., $T \gtrsim 10^{7} \mathrm{~K}$ ) and of low optical thickness, is located above the obscuring torus and electron-scatters the continuum photons coming from the central source (Krolik \& Kallman 1987). If the ionized scattering medium is optically thin, photoelectric absorption is of little importance for the electron-scattered continuum, so the emergent spectrum essentially retains the spectral shape of the primary emission (except for a high-energy cutoff due to Compton down-scattering). This same warm scattering medium most likely produces the higher-energy $\mathrm{Fe}-\mathrm{K} \alpha$ lines from, respectively, He-like iron (Fe XXV) at $\sim 6.7$ and H-like iron (Fe XXVI) at $6.97 \mathrm{keV}$.

A combination of warm and cold reflections is, therefore, a plausible model for the observed X-ray spectra of Sy2 sources hidden in galactic nuclei (Matt et al. 1996, 1997). The relative strength of the cold and warm reflections can possibly be determined from fitting the composite model to 2-10 keV ASCA measurements of the proto-typical Sy2 galaxy NGC 1068. From the fraction of 2-10 keV flux that comes from cold reflection, and an independent estimate of the intrinsic $L_{2-10}^{\prime} \simeq 7 \times$ $10^{43} \mathrm{erg} \mathrm{s}^{-1}$ from the [O III] $\lambda 5007$ luminosity, and a $2-10 \mathrm{keV}$ albedo of 0.022 for the whole inner wall of the torus, Iwasawa et al. (1997) deduced that a fraction $f_{\mathrm{C}} \simeq 4.4 \times 10^{-2}\left(L_{43.8}^{\prime}\right)^{-1}$ of the inner surface of the torus was visible to us. Such a small fraction of the reflecting inner surface of the torus indicates that the torus is viewed nearly edge-on. Iwasawa et al. (1997) further deduced the warm-scattered luminosity fraction to be $f_{\mathrm{w}} \simeq 7.8 \times 10^{-4}\left(L_{43.8}^{\prime}\right)^{-1}$. The warm-to-cold reflection luminosity ratio in NGC 1068 is then 0.018 . For a less extreme inclination of the absorbing torus - i.e. for a higher fraction of the reflecting surface being visible to us - the $2-10 \mathrm{keV}$ spectrum of a Sy2-like source is even more dominated by cold reflection. Thus, we may conclude that the spectral features of cold reflection, i.e. a "flat" $(\Gamma \sim 1.3) 2-10 \mathrm{keV}$ continuum and a $6.4 \mathrm{keV} \mathrm{K} \alpha$ line from "cold" iron, constitute the X-ray signature of a strongly absorbed (i.e., Compton-thick) Sy2-like source. Based on these results, a strategy aimed at unveiling strongly absorbed Active Galactic Nuclei (AGNs) is to make spectral observations of galaxies in the band $2-10 \mathrm{keV}$, and to look for a prominent $6.4 \mathrm{keV}$ line superimposed on a rather flat underlying continuum.

A substantial link between circum-nuclear starbursts (SBs) and AGNs may be established if both occur in the same galaxy. If the SB-driven turbulence in the gas increases the mass inflow rate onto a central black hole, a higher emission from the central engine will ensue (e.g., Veilleux 2001). Since a circumnuclear SB is located farther out than the torus and its obscuring material is distributed quasi-isotropically as seen from the central source, the nuclear source will be further obscured by the SB so that the emerging (cold-reflected) X-ray spectrum will be further hardened by SB-related photoelectric absorption (Fabian et al. 1998). Roughly 50\% of all Sy2 galaxies contain circumnuclear SBs (Gonzales Delgado et al. 2001): these composite objects do tend to be more obscured than those lacking SBs. It is indeed reasonable to conjecture that such extra obscuration is caused by the $\mathrm{SB}$, because star-formation rates
(SFRs) per unit area of $\sim 50-100 M_{\odot} \mathrm{yr}^{-1} \mathrm{kpc}^{-2}$ (typical of the innermost $0.1 \mathrm{kpc}$ in such composite galaxies; see Gonzales Delgado et al. 1998) imply $N_{\mathrm{H}} \sim 10^{24} \mathrm{~cm}^{-2}$ (see Kennicutt 1998). A circum-nuclear SB can also be physically connected to a torus. If the mass inflow rate through the accretion disk is higher than the rate of power dissipation in the disk, its opacity will increase, and the disk will puff up into a geometrically thick torus with a large covering angle (as seen from the central engine). This will quickly block the primary AGN continuum and will result in emission of the fluorescent $\mathrm{Fe}-\mathrm{K} \alpha$ line and flat reflection continuum. Indeed, among the heavily obscured $\left(N_{\mathrm{H}}>10^{24} \mathrm{~cm}^{-2}\right)$ sources studied by Levenson et al. (2002), those that exhibit the largest equivalent widths (EWs) have concentrated circumnuclear SBs, whereas in the only galaxy certainly lacking a $\mathrm{SB}, \mathrm{Mrk} 3$, the $\mathrm{Fe}-\mathrm{K} \alpha$ line is relatively weak $(E W \sim 0.7)$.

Well-known examples of coexisting spectral signatures of star formation and Compton-thick AGNs include cases of both type- 1 and type- 2 view of the central AGN. (These views define Sy1 and Sy2 morphology in the "Unified AGN Model", e.g. Urry \& Padovani 1995.) Examples of the former type include Arp 299, NGC 6240, and NGC 4945: in these objects the absorbing matter is moderately thick to Compton scattering $\left(N_{\mathrm{H}} \sim\right.$ a few $\left.\times 10^{24} \mathrm{~cm}^{-2}\right)$, so the the primary AGN spectrum emerges at (source restframe) energies $E \gtrsim 10 \mathrm{keV}$, within the BeppoSAX observing window (Della Ceca et al. 2002; Ballo et al. 2004; Vignati et al. 1999; Guainazzi et al. 2000). The archetypal example of the latter type is NGC 1068 for which BeppoSAX data support a model envisaging a mixture of both cold and warm reflections of an otherwise unseen primary nuclear continuum, the cold reflection component being the dominant one in the 20-100 keV band (Matt et al. 1997).

NGC 4666 is a starburst galaxy (SBG) seen nearly edge-on (see Table 1). Based on multi-frequency observations, Dahlem et al. (1997) have detected an outflow cone - associated with a galactic superwind - emanating from a central SB of $\sim 3.3 \mathrm{kpc}$ in radius, and having an opening angle of $30^{\circ} \pm 10^{\circ}$. The outflow is traced up to $\sim 7.5 \mathrm{kpc}$ above the disk plane by optical emission line filaments, nonthermal radio continuum emission, and soft X-ray emission from hot gas. The multi-wavelength evidence for enhanced star-formation activity in NGC 4666 can be summarized as follows:

(i) Optical spectroscopy exhibits the kinematic signature of an outflow, and the observed line ratios indicate shockheating [e.g., following SB-related supernova (SN) explosions] as the most likely excitation mechanism for the emission-line gas.

(ii) The existence of a synchrotron radio halo (Sukumar et al. 1988; Dahlem et al. 1997) is strong evidence of ongoing or very recent $\mathrm{SB}$ activity. The radio (energy) index ranges from $\alpha_{\mathrm{r}} \sim 0.8$, measured at small galactocentric radii, to $\alpha \gtrsim 1.3$, measured at large radii. This is proof of the presence, close to the disk, of ongoing injection of fresh relativistic electrons whose lifetime against radiative losses, $t=10^{10}(E / \mathrm{GeV})^{-1}(B / \mu \mathrm{G})^{-2} \mathrm{yr}$, is $t \sim 5-50 \mathrm{Myr}$ (for $B \sim$ $15 \mu \mathrm{G}$ (Dahlem et al. 1997) and $E \sim 1-10 \mathrm{GeV})$, i.e. comparable to or shorter than a typical SB age $\left(t_{\mathrm{SB}} \sim 10^{8} \mathrm{yr}\right)$. 
Table 1. General characteristics of NGC 4666.

\begin{tabular}{crllllllllll}
\hline \hline $\operatorname{Type}^{(a)}$ & $\begin{array}{r}\mathrm{RA}^{(b)} \\
(\mathrm{J} 2000)\end{array}$ & $\begin{array}{l}\operatorname{Dec}^{(b)} \\
(\mathrm{J} 2000)\end{array}$ & $\begin{array}{l}D^{(c)} \\
(\mathrm{Mpc})\end{array}$ & $\begin{array}{l}B_{\mathrm{T}}^{\mathrm{i}, 0(\mathrm{~d})} \\
(\mathrm{mag})\end{array}$ & $\begin{array}{l}\log L_{\mathrm{B}}^{0(e)} \\
\left(L_{\odot}\right)\end{array}$ & $\begin{array}{l}\log N_{\mathrm{H}}^{(f)} \\
\left(\mathrm{cm}^{-2}\right)\end{array}$ & $\begin{array}{l}\log L_{\mathrm{FIR}}^{(g)} \\
\left(L_{\odot}\right)\end{array}$ & $\begin{array}{l}\mathrm{Incl}^{\left({ }^{(h)}\right.} \\
(\mathrm{deg})\end{array}$ & $\begin{array}{l}R_{25}^{\mathrm{i}, 0(i)} \\
(\mathrm{kpc})\end{array}$ & $\begin{array}{l}\log L_{0.5-2}^{(j)} \\
(\mathrm{erg} / \mathrm{s})\end{array}$ & $\begin{array}{l}\log L_{2-10}^{(k)} \\
(\mathrm{erg} / \mathrm{s})\end{array}$ \\
\hline $\mathrm{SABc}$ & $12^{\mathrm{h}} 45^{\mathrm{m}} 09^{\mathrm{s}}$ & $-00^{\circ} 27^{\prime} 38^{\prime \prime}$ & 26.3 & 10.68 & 10.76 & 20.23 & 10.69 & $80^{\circ}$ & 17.5 & 40.20 & 40.40 \\
\hline
\end{tabular}

(a) Morphological type, from $R C 3$ (de Vaucouleurs et al. 1991).

(b) RA(2000) and Dec(2000), from $R C 3$ (de Vaucouleurs et al. 1991).

(c) Distance, from Dahlem et al. (1997).

(d) Total B-magnitude, corrected for Galactic and internal extinction, from RC3 (de Vaucouleurs et al. 1991).

(e) $B$-band luminosity, derived using the quoted distance and $B$-magnitude.

(f) Galactic HI column density (Dickey \& Lockman 1990).

(g) Far-infrared (FIR) luminosity (from Dahlem et al. 1997). The FIR flux is defined (Helou et al. 1985) as a combination of the IRAS 60 $\mu \mathrm{m}$ and $100 \mu \mathrm{m}$ fluxes according to $f_{\mathrm{FIR}} \equiv 1.26 \times 10^{-11}\left(2.58 f_{60}+f_{100}\right) \mathrm{erg} \mathrm{s}^{-1} \mathrm{~cm}^{-1}$, where $f_{60}$ and $f_{100}$ are expressed in Jy.

(h) Inclination angle with respect to line of sight (Dahlem et al. 1998).

(i) 25-B-mag photometric radius, corrected for inclination and Galactic absorption, derived using the angular value given by $R C 3$ (de Vaucouleurs et al. 1991) and the quoted distance.

(j) $0.5-2 \mathrm{keV}$ luminosity, derived using the quoted distance and the data presented in this paper. Not corrected for Galactic absorption.

(k) 2-10 keV luminosity, derived using the quoted distance and the data presented in this paper. Not corrected for Galactic absorption.

Table 2. BeppoSAX and XMM-Newton instrumental parameters.

\begin{tabular}{|c|c|c|c|c|c|c|c|c|c|c|c|c|}
\hline & $\mathrm{FOV}^{a}$ & & & $E_{1}-E_{2}^{b}$ & & & $\Delta E^{c}$ & & & Sensitivit & & \\
\hline \multirow[t]{2}{*}{ SAX } & LECS & MECS & PDS & LECS & MECS & PDS & LECS & MECS & PDS & LECS & MECS & PDS \\
\hline & $37^{\prime}$ & $56^{\prime}$ & $\begin{array}{l}\sim 80^{\prime} \\
\end{array}$ & $0.1-4.5$ & $1.6-10$ & $13-300$ & 0.24 & 0.24 & 9 & $10^{-13}$ & $10^{-13}$ & $2 \times 10^{-11}$ \\
\hline \multirow[t]{2}{*}{$X M M$} & MOS1/2 & PN & & MOS1/2 & PN & & MOS1/2 & PN & & MOS1/2 & PN & \\
\hline & $30^{\prime}$ & $30^{\prime}$ & & $0.3-10$ & $0.5-10$ & & 0.13 & 0.13 & & $7 \times 10^{-15}$ & $7 \times 10^{-15}$ & \\
\hline
\end{tabular}

${ }^{a}$ Diameter of the field-of-view.

${ }^{b}$ Instrument energy range, in $\mathrm{keV}$.

${ }^{c}$ Energy resolution, in $\mathrm{keV}$ (at $6 \mathrm{keV}$ for LECS, MECS, MOS1/2, PN; at $60 \mathrm{keV}$ for PDS).

${ }^{d}$ Flux from point source after $100 \mathrm{ks}$ exposure $\left(\mathrm{erg} \mathrm{cm}^{-2} \mathrm{~s}^{-1}\right)$.

The degree of steepening is just what is expected when the electrons lose energy radiatively.

(iii) At energies <2.4 keV NGC 4666 has been previously observed with ROSAT (Dahlem et al. 1997, 1998); the 4 "-resolution HRI image showed faint diffuse emission extending along the major axis and confined mostly to the disk plane, and two point sources located $\sim 1^{\prime}$ along the minor axis above and below the nuclear region. The spatially integrated PSPC spectrum was fit with a twocomponent model (Dahlem et al. 1998) consisting of a PL + thermal model, with the PL photon index set at $\Gamma=$ 1.9 , and a derived plasma temperature (with the chemical abundances set at the solar value) of $k T=0.31_{-0.12}^{+0.31} \mathrm{keV}$.

In this paper we present the first broad-band $(0.1-50 \mathrm{keV}) \mathrm{spec}-$ tral observations of NGC 4666, obtained using the BeppoSAX and XMM-Newton orbiting telescopes. In Sects. 2 and 3 we describe the data reduction and analysis; in Sect. 4 we describe the spectral fitting, whose implications are discussed in Sects. 5 and 6. Our main results are summarized in Sect. 7. Unless explicitly quoted, all the errors reported in this paper are at the $90 \%$ confidence level for one interesting parameter $\left(\Delta \chi^{2}=2.71\right)$.

\section{Observations and data reduction}

\subsection{BeppoSAX measurements}

NGC 4666 was observed by BeppoSAX with the three narrowfield instruments (NFIs) (see Tables 2 and 3): the Low Energy Concentrator Spectrometer (LECS: Parmar et al. 1997), the Medium Energy Concentrator Spectrometer (MECS: Boella et al. 1997), and the Phoswich Detector System (PDS: Frontera et al. 1997).

The cleaned and linearized data produced by the BeppoSAX Scientific Data Center ${ }^{1}$ (ASDC) were analyzed using standard software packages (XSELECT v1.4, FTOOLS v4.2, and XSPEC v11.2). For the MECS, we used the event file made by merging the data of the two, properly equalized, MECS units. At the spatial resolution of the NFI instruments, NGC 4666 was not resolved. No significant source flux variation was detected over the observing period.

\subsection{XMM-Newton measurements}

NGC 4666 was observed by XMM-Newton on 2002 June 27-28, for a total good exposure of $\sim 50 \mathrm{ks}$ with the European Photon Imaging Camera (EPIC) detectors. Data were taken

\footnotetext{
${ }^{1}$ See http://www.asdc.asi.it/bepposax
} 
Table 3. BeppoSAX and XMM-Newton observation log.

\begin{tabular}{|c|c|c|c|c|c|c|c|}
\hline & Date & Exposure & $\mathrm{ime}^{a}(\mathrm{ks}$ & & Count ra & $b\left(10^{-3}\right.$ & $\left.\mathrm{s}^{-1}\right)$ \\
\hline \multirow{2}{*}{ 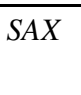 } & 2001 Dec. $14-18$ & LECS & MECS & PDS & LECS & MECS & PDS \\
\hline & & 42.92 & 131.83 & 58.11 & 2.23 & 3.76 & $<18.0$ \\
\hline \multirow{2}{*}{$X M M$} & 2002 June $27-28$ & MOS $1 / 2$ & PN & & MOS $1 / 2$ & PN & \\
\hline & & 57.1 & 48.3 & & $\sim 0.013$ & 0.04 & \\
\hline
\end{tabular}

${ }^{a}$ On-source net exposure time. The LECS exposure time is considerably shorter than that of the MECS, because the LECS could operate only when the spacecraft was not illuminated by the Sun.

${ }^{b}$ Background-subtracted source count rates, with photon counting statistics errors. For the PDS the $90 \%$ upper limit is given.

with the EPIC PN detector (Strüder et al. 2001) in extended full-frame mode, and with the EPIC metal oxide semiconductor (MOS) detectors (Turner et al. 2001) in full-window mode (see Tables 2 and 3). The data were reduced using version 5.4 .1 of the XMM-SAS software, using the standard processing scripts (emproc and epproc). 10-12 keV light curves were inspected and found to be at a nominal rate for the whole observation. Data were selected using event patterns 0-12 (for the MOS) and pattern 0-4 (for the PN), and only good X-ray events (using the selection expression "FLAG $=0$ " in evselect) were included.

Images obtained with the PN detector and with the MOS1 are shown in Fig. 1. These clearly show that NGC 4666 is extended in both energy bands. The extension is most prominent at the lower energy band, and seems to originate in a truly diffuse emission. At higher energies, the emission is primarily from point sources, including a nuclear source that is the brightest.

\section{Spectral analysis}

\subsection{BeppoSAX data}

To maximize the statistics and the $s / n$ ratio, the LECS and MECS source counts were extracted from a circular region of $4^{\prime}$ radius. Background counts were extracted from high Galactic latitude "blank" fields (provided by the BeppoSAX ASDC) using an extraction region corresponding in size and detector position to that used for the source. The PDS spectra were extracted with the standard pipeline (with the rise-time correction applied) provided directly by the BeppoSAX ASDC; the simultaneously measured off-source background was used. Significant emission of NGC 4666 was detected by the LECS and MECS, but not by the PDS instrument (see Table 3).

Spectral channels corresponding to energies $0.1-4 \mathrm{keV}$ and 1.8-10 keV respectively have been used for the analysis of the LECS and MECS data (as suggested by the BeppoSAX "Cookbook", see Fiore et al. 1999); PDS data are in the 13-60 keV spectral channels. LECS and MECS source counts were rebinned to have $s / n>3$ in each energy bin; PDS source counts were rebinned to have $s / n>2$. Standard calibration files were used in the fitting procedure; the LECS/MECS and PDS/MECS cross-constants normalizations were allowed to vary in the ranges proposed by the BeppoSAX "Cookbook".
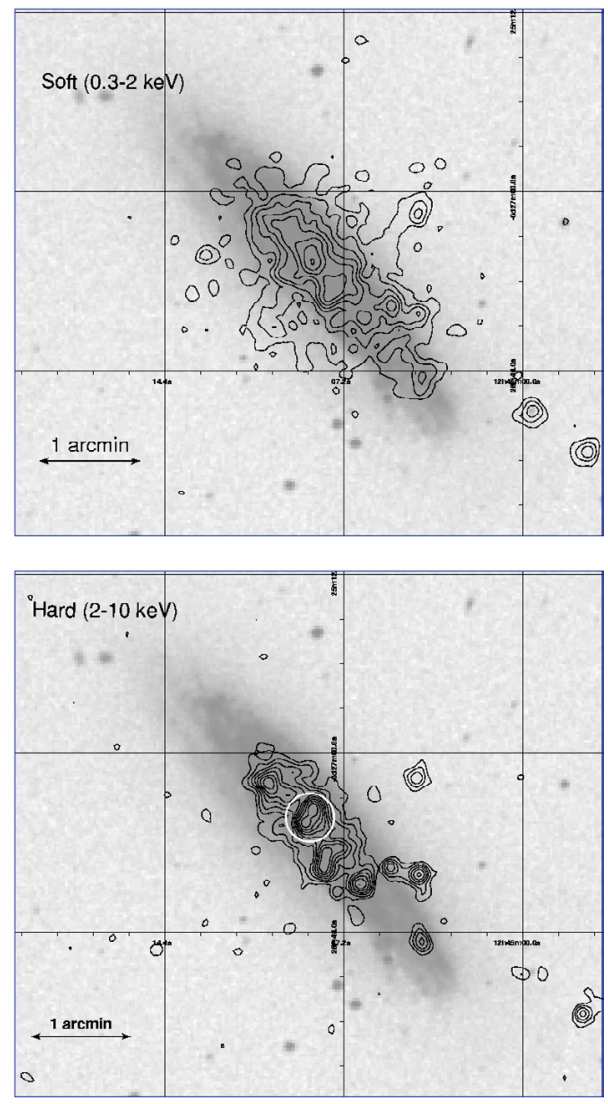

Fig. 1. Soft (top) and hard (bottom) X-ray contours obtained with the MOS1 overplotted on the optical image from the DSS. Contours have been obtained after Gaussian smoothing. They span a surface brightness range of $\sim 0.38-9.4 c \operatorname{arcsec}^{-2}$ in the soft band, and $\sim 0.3-3.6 c \operatorname{arcsec}^{-2}$ in the hard band. In the lower panel, the $15^{\prime \prime}$-radius annulus marks the nuclear extraction region, data from which are shown in Fig. 5.

\subsection{XMM-Newton data}

The nuclear spectrum was extracted (for both MOS and PN detectors) from a circular region of $15^{\prime \prime}$ radius around the nucleus (shown in Fig. 1). Background spectra were extracted from offset circles, close to NGC 4666 but free of any background source. Standard response matrices and ancillary files were used. The background-subtracted spectrum was fitted using XSPEC version v11.2, including $0.3-10 \mathrm{keV}$ data for the MOS and $0.5-10 \mathrm{keV}$ data for the PN. Data were binned so as to have at least 20 counts/bin to allow the use of $\chi^{2}$ 

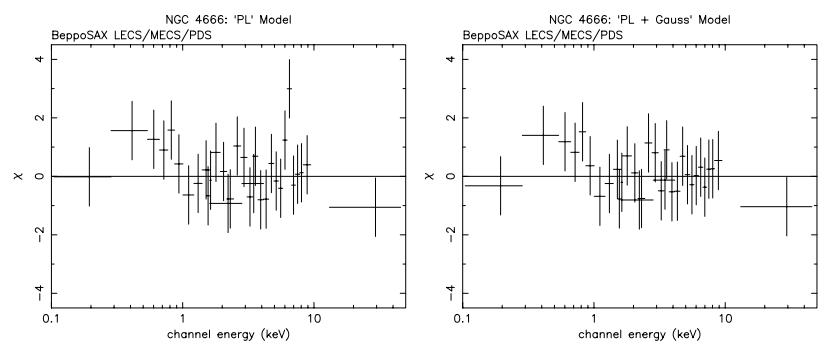

Fig. 2. Left: residuals of the fit of BeppoSAX data to model A (PL with $\Gamma=2.15$ ). Right: residuals of the fit of BeppoSAX data to model $\mathrm{C}$ (PL with $\Gamma=2.27$, plus a Gaussian line at $E=6.46 \mathrm{keV}$ ): the excess at $\sim 6.5 \mathrm{keV}$ has disappeared, but not that at $\lesssim 1 \mathrm{keV}$.

Table 4. Spectral analysis of BeppoSAX data.

\begin{tabular}{ll}
\hline \hline Model & Parameters \\
\hline $\mathbf{A}: \mathrm{pl}$ & $\chi^{2} / v=49.62 / 43$ \\
$\Gamma$ & $2.15 \pm 0.15$ \\
\hline $\mathbf{B}:$ mekal & $\chi^{2} / v=50.24 / 43$ \\
$k T(\mathrm{keV})$ & $4.09 \pm 0.77$ \\
$Z\left(Z_{\odot}\right)$ & $1.0(\mathrm{f})$ \\
\hline $\mathbf{C}: \mathrm{pl}+\mathrm{Gauss}$ & $\chi^{2} / v=38.63 / 41$ \\
$\Gamma$ & $2.27 \pm 0.17$ \\
Line: $\sigma_{\mathrm{E}} ; \mathrm{E}(\mathrm{keV}) ; \mathrm{EW}(\mathrm{keV})$ & $0.0(\mathrm{f}) ; 6.46 \pm 0.55 ; 1.87 \pm 0.60$ \\
\hline $\mathbf{D}: \mathrm{mekal}+\mathrm{pl}+\mathrm{Gauss}$ & $\chi^{2} / v=33.46 / 39$ \\
$k T(\mathrm{keV})$ & $0.23 \pm 0.05$ \\
$Z\left(Z_{\odot}\right)$ & $1.0(\mathrm{f})$ \\
$\Gamma$ & $2.13 \pm 0.19$ \\
Line: $\sigma_{E} ; \mathrm{E}(\mathrm{keV}) ; E W(\mathrm{keV})$ & $0.0(\mathrm{f}) ; 6.48 \pm 0.11 ; 1.66 \pm 0.50$ \\
\hline
\end{tabular}

minimization during the spectral fits. The nuclear spectrum is plotted in Fig. 5 (using $s / n \geq 3$ per bin).

\section{Main spectral components}

The basic model components used in various combinations to fit BeppoSAX data are a PL, a Gaussian line, and the Mekal model describing thermal emission from optically thin hot plasma (see the XSPEC package). We include photoelectric absorption due to Galactic foreground, corresponding to HI column density $N_{\mathrm{H}, \mathrm{Gal}}=1.7 \times 10^{20} \mathrm{~cm}^{-2}$. Also included is intrinsic absorption in NGC 4666 to be determined from the spectral analysis. The results of the spectral analysis are summarized in Table 4.

Single-component models do not provide an adequate description of the BeppoSAX broad-band spectrum of NGC 4666. A single unabsorbed PL model (model A) and a singletemperature thermal model (model B) are rejected at $>99.9 \%$ confidence level. In Fig. 2-left we show the ratio between the data and model A. A broad excess is evident at $\sim 0.5 \mathrm{keV}$, and a line-like excess is seen at $\sim 6.5 \mathrm{keV}$. The residuals around $0.5 \mathrm{keV}$ suggest the presence of a soft thermal component, and the feature at $\sim 6.5 \mathrm{keV}$ suggests $\mathrm{Fe}-\mathrm{K} \alpha$ line emission.

Multi-component models including different combinations of thermal, PL, and Gaussian-line components can then be fitted to the data. A PL+Gaussian-line model (model C), although
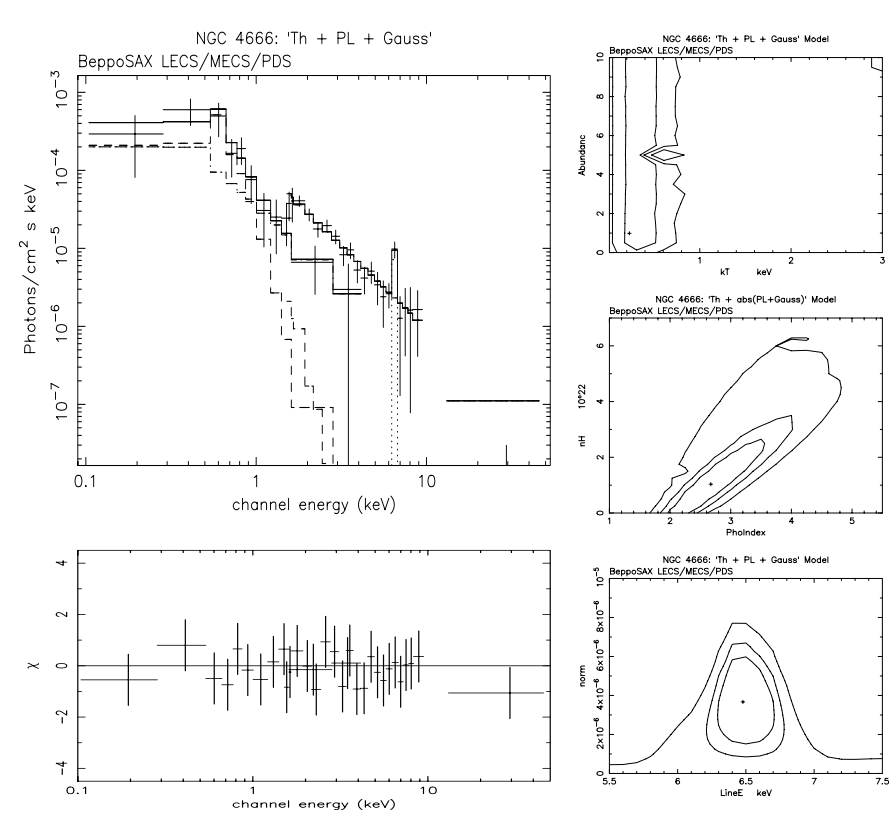

Fig. 3. The model spectrum $\mathrm{C}$, unfolded of instrumental response (top left); and the fit residuals (bottom left). The 68\%, 90\% and 99\% probability contours for two interesting parameters for: (top right) the temperature, $k T$ (in $\mathrm{keV}$ ), and the chemical abundance, $Z$ (in solar units), of the thermal component; (middle right) the intrinsic photon index, $\Gamma$, and the in situ HI column density, $N_{\mathrm{H}}$, for the PL component; and (bottom right) $\mathrm{Fe}-\mathrm{K}$ line energy and normalization.

clearly marking an improvement over models A and B, is still unsatisfactory because it does not remove the excess residuals at $\sim 0.5 \mathrm{keV}$ (Fig. 2-right). A simple model that provides an acceptable statistical fit (model D) includes: $i$ ) an unabsorbed thermal plasma with $k T \simeq 0.23 \pm 0.05 \mathrm{keV}$ (with the chemical abundance set to the solar value); ii) an unabsorbed PL with photon index $\Gamma \simeq 2.1 \pm 0.2$; and iii) a narrow $\left(\sigma_{E} \sim 0\right.$ ) Gaussian line at $E=6.47 \pm 0.12 \mathrm{keV}$, with equivalent width $E W \sim 1.7 \mathrm{keV}$. In Fig. 3 we show model D (top left, with unfolded instrumental response) and the residuals of the fit (bottom left), as well as the confidence countours of some physically meaningful parameter pairs (right). (If we allow for intrinsic absorption, none is deduced in the soft thermal component, while some is found (at $>90 \%$ confidence level) in the PL component, corresponding to $N_{\mathrm{H}}=(1.0 \pm 0.8) \times 10^{22} \mathrm{~cm}^{-2}$. Intrinsic absorption would cause the PL slope to steepen to $\Gamma=2.7 \pm 0.5$; see Fig. 3-middle right.)

The spatially integrated $0.5-10 \mathrm{keV}$ spectra of local SBGs are well fitted by a model including soft $(k T<1 \mathrm{keV})$ thermal emission and a higher-energy component consisting of either $\gtrsim 5 \mathrm{keV}$ thermal emission or a $\Gamma \sim 1.5-2$ PL (e.g., Dahlem et al. 1998). By its continuum properties, therefore, the BeppoSAX spectrum of NGC 4666 seems to be a typical SBG spectrum.

\section{The Fe-K $\alpha$ emission line}

What makes the integrated spectrum of NGC 4666 quite outstanding among known SBG spectra is the huge $(E W \sim 2 \mathrm{keV})$ emission feature clearly required by the BeppoSAX data at 

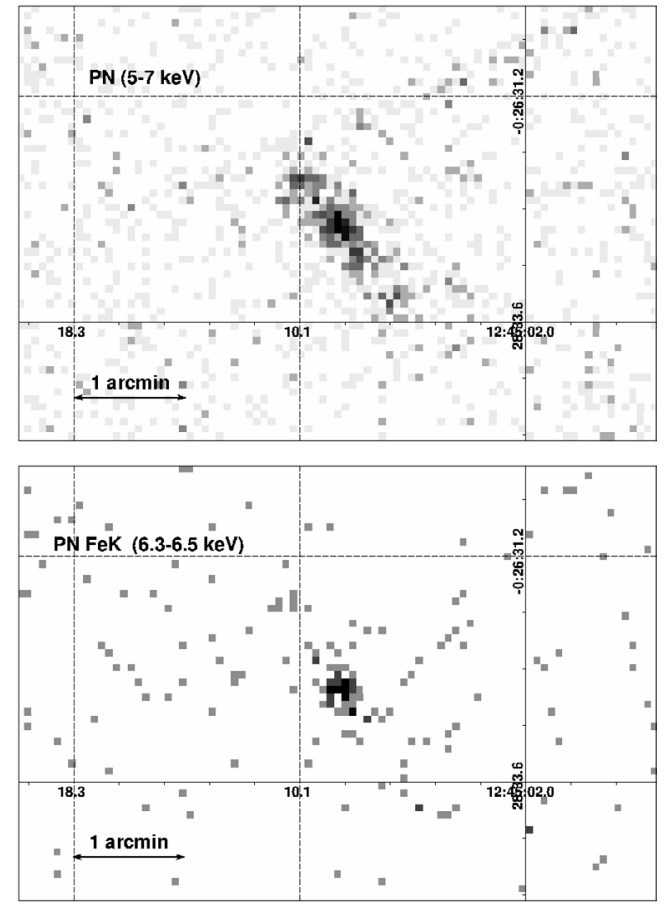

Fig. 4. $\mathrm{PN}$ raw image ( $1 \mathrm{pix}=4 \mathrm{arcsec})$ in the $5-7 \mathrm{keV}$ energy band (top), and in the 6.3-6.5 keV band (bottom).

$\sim 6.5 \mathrm{keV}$ (see Fig. 3 - bottom right). In fact, in SBG spectra emission between 6 and $7 \mathrm{keV}$ is uncommon, inconspicuous $(E W \lesssim 0.3 \mathrm{keV}$ ), and centered at $6.7 \mathrm{keV}$ (and hence it is identified with $\mathrm{K} \alpha$ emission from high-ionization iron - e.g., Persic et al. 1998; Griffiths et al. 2000; Pietsch et al. 2001).

As this emission is unresolved in the BeppoSAX data, we search for it in the archival $X M M$-Newton data. The superior spatial resolution of the latter allows us to identify the line as originating from the nuclear region, and clearly distinguish it from the extended continuum emission (see Fig. 4).

The nuclear location of the line, revealed by XMM-Newton, could be the clue to the presence of a strongly absorbed AGN in NGC 4666. If so, according to the Compton-thick model predictions we expect to observe a prominent $(E W \gtrsim 1 \mathrm{keV})$ line centered at $6.4 \mathrm{keV}$ and superposed on a flat continuum underlying the line itself and cospatial with it.

Spectral analysis of $X M M$-Newton data for the nuclear and circum-nuclear region does indeed show such a line $(E \simeq$ $6.42 \pm 0.02 \mathrm{keV}$ with $E W \simeq 0.7 \pm 0.2 \mathrm{keV})$, superimposed on a flat $(\Gamma \sim 1.3)$ continuum (see Fig. 5 and Table 5$)$. We identify the line as due to the $\mathrm{K} \alpha$ transition at $6.4 \mathrm{keV}$ from low-ionization iron.

To check for consistency of the Compton-thick hypothesis, we try a cold continuum reflection model (PEXRAV in the standard XSPEC spectral fitting package). This model represents the emission that would be expected from, e.g., a circum-nuclear torus that totally blocks the direct view of the primary AGN spectrum (i.e., the type-2 configuration in the unified model of AGNs). As commonly done for standard primary spectra of Sy1 and Sy2 galaxies (e.g. Turner et al. 2000), we assume a PL with photon index $\Gamma=1.9$ for both the primary (completely absorbed) component and the
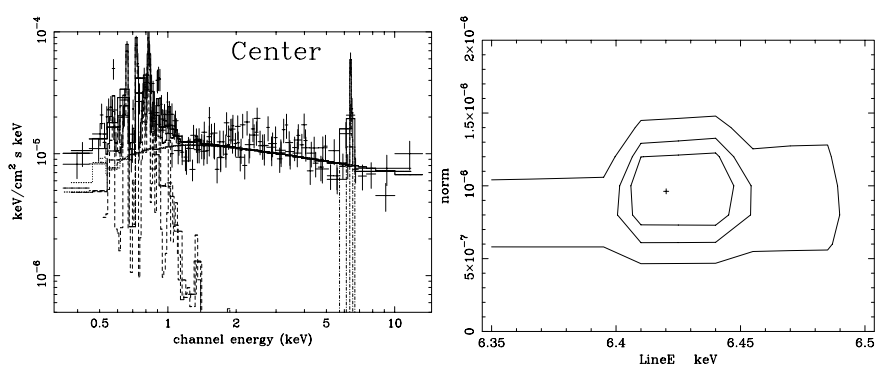

Fig. 5. Left: Spectrum from the nuclear (15" radius) region (see Fig. 1, bottom). A very strong neutral $\mathrm{Fe}-\mathrm{K} \alpha$ line at $6.40 \mathrm{keV}$, and the underlying cospatial flat continuum (approximated by a $\Gamma \simeq 1.3$ PL model), are clearly visible. Right: $68 \%, 90 \%$ and $99 \%$ probability contours for the Fe-K $\alpha$ line's energy versus normalization. The emission line is clearly significant and spectrally localized.

Table 5. Spectral analysis of XMM-Newton data.

\begin{tabular}{ll}
\hline \hline Model & Parameters \\
\hline $\mathbf{A}:$ mekal+pl+Gauss & $\chi^{2} / v=220.05 / 163$ \\
$k T(\mathrm{keV})$ & $0.33 \pm 0.02$ \\
$Z\left(Z_{\odot}\right)$ & $1.0(\mathrm{f})$ \\
$\Gamma$ & $1.31 \pm 0.05$ \\
Line: $\sigma_{E} ; E(\mathrm{keV}) ; E W(\mathrm{keV})$ & $0.0(\mathrm{f}) ; 6.42 \pm 0.05 ; 0.75 \pm 0.25$ \\
\hline $\mathbf{B}:$ mekal+pl+Gauss+refl. & $\chi^{2} / v=236.35 / 163$ \\
$k T(\mathrm{keV})$ & $0.32 \pm 0.02$ \\
$Z\left(Z_{\odot}\right)$ & $1.0(\mathrm{f})$ \\
$\Gamma$ & $1.9(\mathrm{f})$ \\
$R(=\mathrm{refl} . / \mathrm{dir})$. & $6 \pm 2$ \\
Line: $\sigma_{E} ; E(\mathrm{keV}) ; E W(\mathrm{keV})$ & $0.0(\mathrm{f}) ; 6.42 \pm 0.05 ; 0.75 \pm 0.25$ \\
\hline
\end{tabular}

warm-scattered component (which is visible in the $\sim 1-5 \mathrm{keV}$ band). We also assume solar chemical abundances, full ionization of $\mathrm{H}$ and $\mathrm{He}$, and a viewing angle of $80^{\circ}$ (i.e., we take the torus axis to coincide with the galaxy spin axis). The resulting fit of this (astrophysically motivated) model is satisfactory (see Table 5), similar to the previous case of the (heuristic) singlePL model. The $2-10 \mathrm{keV}$ flux of the best-fit cold-reflection component is $F_{\text {refl }}^{\mathrm{AGN}} \sim 4.8 \times 10^{-14} \mathrm{erg} \mathrm{cm}^{-2} \mathrm{~s}^{-1}$, yielding an observed luminosity of $L_{\text {refl }}^{\mathrm{AGN}} \sim 4.0 \times 10^{39} \mathrm{erg} \mathrm{s}^{-1}$ (see Fig. 5). If we assume - as did Iwasawa et al. (1997) - that the intrinsic luminosity of the hidden AGN is a factor $\gtrsim 50$ larger than the observed value, then $L_{\text {intr }}^{\mathrm{AGN}} \gtrsim 2 \times 10^{41} \mathrm{erg} \mathrm{s}^{-1}$.

It should be emphasized that the extraction radius $\left(\sim 15^{\prime \prime}\right)$ corresponds to a linear size of $1.9 \mathrm{kpc}$. Thus, this large circumnuclear region includes a substantial fraction of the SB emission. This may well explain why the continuum underlying the $6.4 \mathrm{keV}$ line is somewhat steeper than predicted by a pure Compton-thick model (see Fig. 5).

\section{Origin of the continuum}

\subsection{Thermal component}

The thermal plasma component identified in the spatially integrated BeppoSAX data agrees (within errors) with the 
earlier finding (based on ROSAT data) by Dahlem et al. (1998). Our BeppoSAX data are not sensitive enough to determine the chemical abundance, whereas the temperature is better constrained to $k T \sim 0.3 \pm 0.1 \mathrm{keV}$ for a broad range of the abundance (see Fig. 3 - upper right); also, the temperature is decoupled from the slope of the PL component.

Thermal emission is usually interpreted as arising from the interaction between the outgoing galactic wind and the ambient ISM. The wind is hot but very tenuous, while the ISM is dense but cold, so none of these is likely to emit appreciably in the X-ray region. The boundary region between the two phases, however, does achieve a combination of temperature and density suitable for sub-keV emission from a few percent of the wind mass (Strickland \& Stevens 2000; Suchkov et al. 1996). Single-temperature models $(k T \sim 0.8 \mathrm{keV})$ and sometimes two-temperature models (with $k T \sim 0.8$ and $\sim 0.3 \mathrm{keV}$ ) have been deduced from ASCA + ROSAT (e.g., Dahlem et al. 1998) and XMM-Newton (Franceschini et al. 2003) data of actively star-forming galaxies ${ }^{2}$.

Analysis of archival XMM-Newton data shows that the soft $(0.3-2 \mathrm{keV})$ emission is diffuse and essentially co-extended with the higher energy $2-10 \mathrm{keV}$ emission (see Fig. 1). Because the soft emission is largely dominated by the thermal component, which arises from the galactic winds that are driven by current SF activity, its spatial distribution suggests that the SB is occurring throughout the disk in NGC 4666.

\subsection{PL component}

Persic \& Rephaeli (2002; hereafter, PR02) have quantitatively assessed the roles of the various X-ray emission mechanisms in star-forming galaxies. They have used an equilibrium stellarpopulation synthesis model of the Galactic population of highmass X-ray binaries (HMXBs) and low-mass X-ray binaries (LMXBs). The abundance of SN remnants (SNRs), from both Type II and Type Ia events, was also consistently estimated. From the literature PR02 derived typical spectra for these source classes. The spectral properties and relative abundances of the various classes of stellar sources allowed PR02 to calculate the composite X-ray spectrum arising from a stellar population of Galactic composition.

PR02 determined that the $\sim 2-15 \mathrm{keV}$ emission is dominated by LMXBs; this is not surprising, given that the synthetic spectrum was calibrated by that of a normal, quietly star forming galaxy like ours. Due to the long times between the formation and the onset of the X-ray phase (the optical companion is a subsolar main-sequence star) in LMXBs, their emission traces the higher, past Galactic SFR, and not the lower, current SFR. Thus in order to obtain an estimate of the current SFR, the spectral contribution of LMXBs should not be included.

Recent observations of nearby galaxies - mostly with Chandra - have revealed two new populations of X-ray point sources (XPs), not present in our Galaxy, with 2-10 keV

${ }^{2}$ Part of the measured thermal emission may also come from OB stars, which are expected to be abundant in SB regions and whose thermal emission is characterized by $k T \sim 0.2 \mathrm{keV}, L \sim 10^{33} \mathrm{erg} \mathrm{s}^{-1}$ (see PR02). luminosities in the range $2 \times 10^{38} \mathrm{erg} \mathrm{s}^{-1} \leq L_{2-10} \leq 10^{39} \mathrm{erg} \mathrm{s}^{-1}$ (Very Luminous Sources: VLXs), and with $L>10^{39} \mathrm{erg} \mathrm{s}^{-1}$ (Ultra-Luminous Sources: ULXs) (Fabbiano \& White 2003) $^{3}$. In the 2-10 keV band relevant here, mean VLX and ULX spectra are best-fit by PLs with photon indices of $\sim 2$ and $\sim 1.2$, reminiscent of, respectively, Galactic Black-Hole-X-ray binaries (BHXBs) and $\mathrm{HMXBs}^{4}$ (e.g., Zezas et al. 2002; Terashima \& Wilson 2004; Foschini et al. 2002; see also Fabbiano \& White 2003). VLXs and ULXs may describe different luminosity/spectral states of BHXBs as a function of the mass infall rate onto the $\mathrm{BH}^{5}$. The discovery of these sources has augmented the variety of known stellar endproducts that emit X-rays, and the PR02 method can easily incorporate such new classes (Persic \& Rephaeli 2004).

The population of VLX and ULX sources in a galaxy is more abundant the more active is the star formation in that galaxy. Specifically, if the integral XP luminosity function (XPLF) is described as $N(>L) \propto L^{-\alpha}$ (which corresponds to a differential XPLF of the type $\left.\frac{\mathrm{d} N}{\mathrm{~d} L} \propto L^{-(1+\alpha)}\right)$, based on a wealth of Chandra oservations it has been found that XPLFs are less steep in SFGs than in normal spirals and ellipticals. (For example, the two most nearby SB galaxies have $\alpha \sim 0.5$ (M 82) and $\alpha \sim 0.8$ (NGC 253), while normal spirals have $\alpha \sim 1.2$, and ellipticals have approximately $\alpha \geq 1.4$; see Kilgard et al. 2002.) So the integrated point-source luminosity of SFGs is dominated by the highest- $L$ sources (Colbert et al. 2004). If the XPLF in NGC 4666 is similar to those in other star-forming galaxies (e.g., $\alpha \sim 0.5-0.8$; see Kilgard et al. 2002), then its spatially integrated $2-10 \mathrm{keV}$ luminosity and spectrum will be dominated by VLXs and ULXs (unless there is a substantial diffuse hard component). The resulting spectral index, $\bar{\Gamma}$, will be $1.2 \lesssim \bar{\Gamma} \lesssim 2$, with the exact value depending on the XPLF index $\alpha$ and on $\Gamma=\Gamma(L)$. Data with angular resolution at the arcsec level and sensitivity at the $\sim 10^{-15} \mathrm{erg} \mathrm{cm}^{-2} \mathrm{~s}^{-1}$ level (e.g., Chandra data) will resolve XPs in NGC 4666 down to $L_{2-10} \sim 5 \times 10^{37} \mathrm{erg} \mathrm{cm}^{-2} \mathrm{~s}^{-1}$. The fluxes and - where possible - the spectra obtained for these XPs would allow one to obtain

\footnotetext{
${ }^{3}$ The two quoted limits correspond to Eddington luminosities for, respectively, a $1.5 M_{\odot}$ neutron star and a $8 M_{\odot}$ black hole $(\mathrm{BH})$ which is the limiting $\mathrm{BH}$ mass obtainable via ordinary stellar evolution. ULXs are called "super-Eddington sources".

${ }^{4}$ In a few cases the optical counterparts of ULX have been identified as O stars (Liu et al. 2002; Roberts et al. 2002), thus suggesting a relation between ULXs and HMXBs, which of course are young objects that trace the current SFR.

${ }^{5}$ Qualitatively, the inverse correlation between $L_{2-10}$ and $\Gamma$ (i.e., flatter spectra for higher luminosities) can be understood within the standard accretion model devised to explain the high $L_{\mathrm{x}}$ of interacting binaries. The increase in $L_{\mathrm{x}}$ is driven by an increase of $\dot{M}$, which in turn means a piling up of material around the emission region that leads to a higher Compton scattering optical depth, and hence to a flatter spectrum. E.g., according to Sunyaev \& Titarchuk (1980) and Shapiro et al. (1976), if a source of photons is embedded in a plasma cloud of optical depth $\tau$ and temperature $T$, the escaping radiation has a high-energy photon index
}

$\Gamma=\left[\frac{9}{4}+\frac{\pi^{2} m_{\mathrm{e}} c^{2}}{3(\tau+2 / 3)^{2} k T}\right]^{1 / 2}-\frac{1}{2}$ 


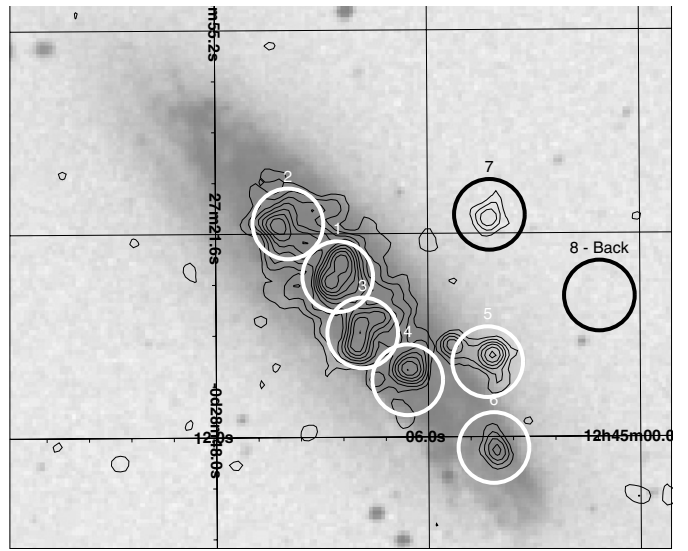

Fig. 6. 2-10 keV contours obtained with the MOS1 overplotted on the optical image from the DSS (for details see Fig. 1). The seven "sources" (i.e., maxima of emission) are identified by $15^{\prime}$-radius circles. An eighth circle denotes an empty field that was used for background estimation.

some firmer clues on their nature. A full analysis of the maxima of emission seen in the $2-10 \mathrm{keV}$ XMM-Newton map is beyond the scope of this paper. A preliminary analysis is, however, in order.

From the $2-10 \mathrm{keV}$ isophotes overlaid on the optical image of NGC 4666 (see Fig. 6), 7 emission maxima can be identified by visual inspection. In order to estimate the fluxes from these locations, we have constructed 7 circles, with radii of $15^{\prime \prime}$, centered on the selected features (so as to include $\geq 70 \%$ of the encircled energy). An eighth, off-galaxy circle was used to estimate the background. Assuming for each "source" a flat spectral shape $(\Gamma=1.3)$ in order to obtain a (likely) upper limit to its $2-10 \mathrm{keV}$ flux and a background as given by the off-galaxy empty field, the most obvious conclusion was that "sources" \#1-4 qualified as ULXs and "sources" \#5-7 qualified as VLXs. However, of such sources in Fig. 6, three quite clearly show structure: \#1, \#3 and \#6. These emissions can be interpreted as resulting from (at least) 2, 3, and 2 sources each, respectively. We know that about half of emission \#1 is due to "cold reflection" from a deeply absorbed type-2 AGN $\left(L_{2-10}^{\mathrm{AGN}, \text { refl }} \sim 0.40 \times 10^{40} \mathrm{erg} \mathrm{s}^{-1}\right.$, see Sect. 5): the remaining flux, corresponding to $L_{2-10} \sim 3.8 \times 10^{39} \mathrm{erg} \mathrm{cm}^{-2} \mathrm{~s}^{-1}$, can be attributed to an ULX. Emission \#3 may be composed of 3 VLXs with $L_{2-10} \sim 10^{39} \mathrm{erg} \mathrm{cm}^{-2} \mathrm{~s}^{-1}$ each. Finally, the elongated emission \#6 may come from 2 VLXs with $L_{2-10} \sim$ $5 \times 10^{38} \mathrm{erg} \mathrm{cm}^{-2} \mathrm{~s}^{-1}$ each. Furthermore, emission \#2 looks quite confused and its isophotes, which tend to become more triangular for decreasing levels of emission, may suggest the presence of three unresolved sources: given the global level of emission measured for this feature, such sources could be (e.g.) 2 VLXs with $L_{2-10} \lesssim 10^{39} \mathrm{erg} \mathrm{s}^{-1}$ each, and 1 ULX with $L_{2-10} \gtrsim 10^{39} \mathrm{erg} \mathrm{s}^{-1}$.

Based on these considerations, we tentatively identify the XPs hinted at by the 2-10 keV XMM-Newton image as being 3 ULXs (with luminosities $\left.2 \lesssim L_{2-10} /\left(10^{39} \mathrm{erg} \mathrm{s}^{-1}\right) \lessgtr 4\right)$ and 9 VLXs (with luminosities $0.5 \lesssim L_{2-10} /\left(10^{39} \mathrm{erg} \mathrm{s}^{-1}\right) \lesssim 2$ ). Collectively, they contribute $1.6 \times 10^{40} \mathrm{erg} \mathrm{cm}^{-2} \mathrm{~s}^{-1}$ in the $2-10 \mathrm{keV}$ band. Adding the estimated cold reflection from the hidden AGN, the total XP luminosity of NGC 4666 becomes $L_{2-10}^{\mathrm{XP}}=2 \times 10^{40} \mathrm{erg} \mathrm{s}^{-1}$, i.e. $80 \%$ of the total $2-10 \mathrm{keV}$ luminosity observed with BeppoSAX (the latter being $L_{2-10}=$ $2.5 \times 10^{40} \mathrm{erg} \mathrm{cm}^{-2} \mathrm{~s}^{-1}$, see Table 1 ). The remaining $20 \%$, missed by the XP luminosity computed from the XMM-Newton map as described above, might be genuinely diffuse (see below), or it could be due to unresolved sources of lower luminosity (i.e., with $L_{2-10} \lesssim 5 \times 10^{38} \mathrm{erg} \mathrm{s}^{-1}$ ), or a combination of the two.

We can gain an insight into this issue by the following argument. If the differential XPLF is of the type $\frac{\mathrm{d} N}{\mathrm{~d} L} \propto L^{-(1+\alpha)}$, our tentative identification of XPs suggests $\alpha \sim 0.5$ (similar to what is observed in M 82, see Kilgard et al. 2002): such an XPLF implies that the collective observed luminosity due to our tentative XPs amounts to $76 \%$ of the XP luminosity integrated down to $10^{38} \mathrm{erg} \mathrm{s}^{-1}$ (i.e. down to the lower luminosity limit defining VLXs). This simple model implies that the XP luminosity, integrated between $10^{38} \mathrm{erg} \mathrm{s}^{-1}$ and $4 \times 10^{39} \mathrm{erg} \mathrm{s}^{-1}$, is $L_{2-10}^{\mathrm{XP}}=(1 / 0.76 \times 1.6+0.4=2.5) \times 10^{40} \mathrm{erg} \mathrm{s}^{-1}$. This matches the integrated 2-10 keV luminosity seen with BeppoSAX. What about the spectral shape? If we assume VLXs to have $\Gamma=$ 2.0-2.5 and ULXs to have $\Gamma=1.3$, our toy model implies $\bar{\Gamma} \simeq 1.7-2.0$, in reasonable agreement with the BeppoSAX result $\bar{\Gamma}=2.1 \pm 0.2$. Therefore a toy model for the XP population, that involves ULXs and VLXs distributed according to an $\alpha=0.5$ XPLF plus a Compton-thick AGN, can bring BeppoSAX data and XMM-Newton data into reasonable agreement as far as both the luminosity and spectral properties are concerned.

That the $2-10 \mathrm{keV}$ emission of NGC 4666, once the AGN emission has been subtracted, is indeed mostly related to current SF activity can be checked as follows. From a sample of SB-powered ultra-luminous IR galaxies (ULIRGs; Franceschini et al. 2003) it can be deduced that the $2-10 \mathrm{keV}$ to FIR emission ratio of pure SBs is $\theta \equiv \log \left(L_{2-10} / L_{\mathrm{FIR}}\right) \sim$ $-4.3 \pm 0.3$ (Persic et al. 2004). Upon subtraction of the AGNrelated emission, NGC 4666 has $\theta \sim-4.0$ : so its disk $2-10 \mathrm{keV}$ emission is roughly at the level implied by its current SFR. This deduction seems reasonable because NGC 4666 exhibits the "smoking gun" proof of being a global SB: a large-scale supergalactic wind and a substantial radio halo. It should also be recalled that NGC 4666 is a member of a small interacting group of galaxies (García 1993); such a dense environment may well have tidally triggered global SF activity in NGC 4666.

\subsubsection{Compton scattered radiation}

As is well known, Compton scattering of (the radiosynchrotron emitting) relativistic electrons by the FIR and cosmic microwave background (CMB) radiation fields results in a PL component with roughly the same photon index, $\Gamma \sim 1.8$, as that of the radio emission (Schaaf et al. 1989; Rephaeli et al. 1991; Dahlem et al. 1997; Sukumar et al. 1988). This closely resembles the observed $2-10 \mathrm{keV}$ spectral profile. Compton emission may contribute $\$ 20 \%$ of the observed 2-10 keV flux, as suggested by the following argument. (Formally, this is the level of $2-10 \mathrm{keV}$ emission needed 
to supplement $L_{2-10}^{\mathrm{XP}}$ (as measured by XMM-Newton) in order to reproduce $L_{2-10}$ (as measured by BeppoSAX).) If the relativistic electrons and seed FIR photons are cospatial with a uniform magnetic field $B$, the Compton luminosity can be estimated from $L_{\mathrm{c}}=L_{\mathrm{s}} U_{\mathrm{ph}} / U_{B}$, where $L_{\mathrm{s}}$ is the total synchrotron luminosity, $U_{\mathrm{ph}}$ is the energy density in the seed photon field, and $U_{B}$ is the energy density in the magnetic field. Integrating the radio spectrum over the $0.01-200 \mathrm{GHz}$ range (the upper limit of the integration contributes very little to the integral), we get $L_{\mathrm{s}} \simeq 5.1 \times 10^{39} \mathrm{erg} \mathrm{s}^{-1}$. For a spherical region of radius $r$, the seed photon energy density is $U_{\mathrm{ph}}=3 L_{\mathrm{bol}} /\left(4 \pi r^{2} c\right)$. Assuming $L_{\mathrm{bol}} \simeq L_{\mathrm{FIR}}$ and $r \simeq 3 \mathrm{kpc}$ (see Dahlem et al. 1997),

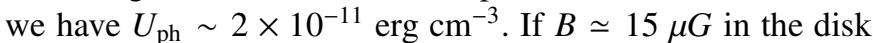
where the FIR emission originates (Dahlem et al. 1997), then $U_{\mathrm{B}}=B^{2} /(8 \pi) \sim 10^{-11} \mathrm{erg} \mathrm{cm}^{-3}$. The Compton luminosity of NGC 4666, integrated over the entire range of upscattered energies is expected to be $L_{\mathrm{c}} \sim 10^{40} \mathrm{erg} \mathrm{s}^{-1}$, suggesting that FIRCompton emission may account for a non-negligible fraction of the observed 2-10 keV emission, $L_{2-10}=2.5 \times 10^{40} \mathrm{erg} \mathrm{s}^{-1}$. However, this estimate depends sensitively on the mean value of $B$ which is not known very well. The corresponding estimate of emission from Compton scattering off the CMB (mostly) in the halo is even less secure, due to substantial uncertainty in the mean halo value of $B$ (even ignoring pertinent spatial considerations), but is likely to be weaker than that of the disk.

\section{Conclusions}

The X-ray spectrum of the nearly edge-on superwind galaxy NGC 4666 was measured in the $0.1-10 \mathrm{keV}$ band with the LECS, MECS, and PDS instruments aboard BeppoSAX, as well as in the $0.3-10 \mathrm{keV}$ band with the EPIC instrument aboard $X M M-N e w t o n$. As seen by BeppoSAX, the emission is not spatially resolved, and significant detection occurs only at energies $<10 \mathrm{keV}$. Emission measured by XMM-Newton is spatially resolved as coming from the whole disk.

SB and AGN activities coexist in NGC 4666. The former, which extends over most of the disk, is revealed, in the $\mathrm{X}$-ray region, by (i) diffuse thermal emission associated with SN-powered galactic wind, and by (ii) high-luminosity XPs that are well-known indicators of enhanced star-formation activity. It is also indicated by (iii) an XPLF index $\alpha \sim 0.5$, suggested here by a preliminary XP analysis of the XMM-Newton data and similar to those derived for other actively star-forming galaxies. The AGN activity is revealed by a prominent $\mathrm{K} \alpha$ line from "cold" iron at $6.40 \mathrm{keV}$ and its relatively flat underlying continuum, both coming from the nuclear region, that originate from the reflection of the primary continuum by the cold inner wall of the circumnuclear torus.

More specifically, our results can be summarized as follows.

(a) At energies $\lesssim 1 \mathrm{keV}$ the spectrum of NGC 4666 is dominated by a $\sim 0.25 \mathrm{keV}$ diffuse thermal plasma component (of unconstrained chemical abundance), distributed throughout the disk, which traces the interaction between the hot, tenuous wind elements and the cool, denser ISM in the disk. The distribution of this sub-keV plasma shows that the current SB phase involves the whole galaxy.

(b) At energies $\sim 2-10 \mathrm{keV}$ the integrated spectrum is dominated by a steep $(\Gamma \sim 2)$ PL component. This emission is probably due to unresolved XPs distributed according to a luminosity function with (differential) index $\alpha \sim 0.5$, and having $L_{2-10} \geq 10^{38} \mathrm{erg} \mathrm{s}^{-1}$. These sources, which are likely to be accreting binaries with $\leq 8 M_{\odot}$ black hole components, are known to dominate the XP luminosity of nearby star-forming galaxies and in the relevant energy range their spectra have $\Gamma \sim 2$ PL (for $0.2 \leq$ $L_{2-10} /\left(10^{39} \mathrm{erg} \mathrm{s}^{-1}\right) \leq 1$ ) and $\Gamma \sim 1.3 \mathrm{PL}$ (for $L_{2-10}>$ $\left.10^{39} \mathrm{erg} \mathrm{s}^{-1}\right)$. A $\Gamma \sim 1.8 \mathrm{PL}$ contribution from Compton scattering of relativistic electrons (whose observed radio index is $\alpha_{\mathrm{r}} \sim 0.8$ ) by the ambient FIR photons may add a truly diffuse $2-10 \mathrm{keV}$ emission.

(c) The prominent $(E W \sim 2 \mathrm{keV})$ emission line, seen but not resolved in the BeppoSAX data, is identified in the XMM-Newton data as fluorescent neutral $\mathrm{Fe}-\mathrm{K} \alpha$ at $6.40 \mathrm{keV}$ originating from the nucleus of NGC 4666. This, together with the presence of a flat $(\Gamma \sim 1.3)$ continuum in the same nuclear region that emits the line, suggests the existence of a strongly absorbed (Compton-thick) AGN, whose intrinsic $2-10 \mathrm{keV}$ luminosity is $L_{2-10} \gtrsim$ $2 \times 10^{41}$ erg s$^{-1}$.

Compared with other composite AGN/SB galaxies, NGC 4666 shows an important difference. For example, in NGC 6240 and Arp 299 the AGNs have high intrinsic X-ray luminosities $\left(\sim 10^{43}-10^{44} \mathrm{erg} \mathrm{s}^{-1}\right)$ and are seen according to the type-1 view (Vignati et al. 1999; Della Ceca et al. 2002). In these galaxies the AGN dominates the emission at $E \gtrsim 2 \mathrm{keV}$ : (a) the highlyabsorbed primary continuum, transmitted through an obscuring cold medium, emerges from the absorber and dominates the emission at $E \gtrsim 10 \mathrm{keV}$; and $(b)$ the unabsorbed primary continuum, scattered into the line-of-sight by a warm gas located outside the absorbing medium, dominates at $2 \mathrm{keV} \lesssim$ $E \lesssim 10 \mathrm{keV}$. Only at $E \sim 1 \mathrm{keV}$ can the SB be recognized from the thermal signatures of the wind components. (A similar situation is encountered in composite AGN/SB-powered ULIRGs: Franceschini et al. 2003 and Persic et al. 2004). The situation in NGC 4666 is very different. Here the AGN has a much lower (estimated) intrinsic luminosity $\left(\sim 2 \times 10^{41} \mathrm{erg} \mathrm{s}^{-1}\right)$ and is seen in type- 2 orientation (the galaxy is edge-on). The combination of these two factors makes the AGN contribution to the $2-10 \mathrm{keV}$ emission of NGC 4666 quite small, $15 \%$, so the $\mathrm{SB}$ - far from being outshone by the AGN - dominates the integrated $0.3-10 \mathrm{keV}$ continuum.

The case that a low-luminosity type- 2 AGN is identified in the middle of the overwhelming glare of a SB makes NGC 4666 an observationally interesting and quite rare case. This occurrence owes to the simultaneous use of satellite data having widely different characteristics. The low-resolution BeppoSAX data have revealed the dominant continuum component integrated over the disk, thereby giving information on the disk-wide SB; they have also revealed the existence of a prominent $6.4 \mathrm{keV}$ line, apparently unrelated to the observed continuum. The higher-resolution XMM-Newton data have shown 
that the $6.40 \mathrm{keV} \mathrm{Fe}-K \alpha$ line originates from the central region: a close-up view of such region has revealed a flat $(2-10 \mathrm{keV})$ local continuum, which indicates the existence of a Comptonthick type-2 AGN; the XMM-Newton data have further revealed a number of possible $X$-ray point sources that, intepreted as accretion-powered binary systems associated with active ongoing star formation, can naturally explain the integrated continuum observed by BeppoSAX.

Acknowledgements. We have analyzed observations obtained with $X M M-N e w t o n$, an ESA science mission with instruments and contributions directly funded by ESA Member States and NASA (USA). This research has made use of the NASA/IPAC Extragalactic Database (NED) which is operated by the Jet Propulsion Laboratory, California Institute of Technology, under contract with NASA. We acknowledge the BeppoSAX SDC team for providing pre-processed event files. We thank an anonymous referee for his/her suggestions that led to a very substantial improvement of the paper.

\section{References}

Antonucci, R. R. J. 1993, ARA\&A, 31, 473

Antonucci, R. R. J., \& Miller, J. S. 1985, ApJ, 297, 621

Ballo, L., Braito, V., Della Ceca, R., et al. 2004, ApJ, 600, 634

Boella, G., Chiappetti, L., Conti, G., et al. 1997, A\&AS, 122, 327

Colbert, E. J. M., Heckman, T. M., Ptak, A. F., \& Strickland, D. K. 2004, ApJ, 602, 231

Dahlem, M., Petr, M., Lehnert, M. D., et al. 1997, A\&A, 320, 731

Dahlem, M., Weaver, K. A., \& Heckman, T. M. 1998, ApJS, 118, 401

de Vaucouleurs, G., de Vaucouleurs, A., Corwin, H. G. Jr., et al. 1991, The Third Reference Catalogue of Bright Galaxies (Austin: Univ. Texas Press) (RC3)

Della Ceca, R., Ballo, L., Tavecchio, F., et al. 2002, ApJ, 581, L9

Dickey, J. M., \& Lockman, F. J. 1990, ARA\&A, 28, 215

Fabbiano, G., \& White, N. E. 2003, in Compact Stellar X-Ray Sources, ed. W. Lewin, \& M. van der Klis (Cambridge University Press) [arXiv: astro-ph/0307077]

Fabian, A. C., Barcons, X., Almaini, O., \& Iwasawa, K. 1998, MNRAS, 297, L11

Fiore, F., Guainazzi, M., Grandi, P. 1999, Handbook for NFI Spectral Analysis available at:

ftp://sax.sdc.asi.it/pub/sax/doc/software docs/ saxabc v1.2.ps.gz

Foschini, L., Di Cocco, G., Ho, L. C., et al. 2002, A\&A, 392, 817

Franceschini, A., Braito, V., Persic, M., et al. 2003, MNRAS, 343, 1181

Frontera, F., Costa, E., Dal Fiume, D., et al. 1997, A\&AS, 122, 357

García, A. M. 1993, A\&AS, 100, 47
George, I. M., \& Fabian, A. C. 1991, MNRAS, 249, 352

Gonzales Delgado, R. M., Heckman, T., \& Leitherer, C. 2001, ApJ, 546, 845

Gonzales Delgado, R. M., Heckman, T., \& Leitherer, C., et al. 1998, ApJ, 505, 174

Griffiths, R. E., Ptak, A., Feigelson, E. D., et al. 2000, Science, 290, 1325

Guainazzi, M., Matt, G., Brandt, W. N., et al. 2000, A\&A, 356, 463

Helou, G., Soifer, B. T., Rowan-Robinson, M. 1985, ApJ, 298, L7

Iwasawa, K., Fabian, A. C., \& Matt, G. 1997, MNRAS, 289, 443

Kennicutt, R. C. 1998, ApJ, 498, 541

Kilgard, R. E., Kaaret, P., Krauss, M. I., et al. 2002, ApJ, 573, 138

Krolik, J. H., \& Kallman, T. R. 1987, ApJ, 320, L5

Levenson, N. A., Krolik, J. H., Zycki, P. T., et al. 2002, ApJ, 573, L81

Liu, J.-F., Bregman, J. N., \& Seitzer, P. 2002, ApJ, 580, L31

Matt, G., Brandt, W. N., \& Fabian, A. C. 1996, MNRAS, 280, 823

Matt, G., Guainazzi M., Frontera F., et al. 1997, A\&A, 325, L13

Parmar, A. N., Martin, D. D. E., Bavdaz, M., et al. 1997, A\&AS, 122, 309

Persic, M., Mariani, S., Cappi, M., et al. 1998, A\&A, 339, L33

Persic, M., \& Rephaeli, Y. 2002, A\&A, 382, 843

Persic, M., \& Rephaeli, Y. 2004, in Proc. 5th INTEGRAL Workshop, in press [arXiv: astro-ph/0403548]

Persic, M., Rephaeli, Y., Braito, V., et al. 2004, A\&A, 419, 849

Pietsch, W., Roberts, T. P., Sako, M., et al. 2001, A\&A, 365, L174

Rephaeli, Y., Gruber, D., Persic, M., \& McDonald, D. 1991, ApJ, 380, L59

Roberts, T. P., Goad, M. R., Ward, M. J., et al. 2002, in New Visions of the X-ray Universe in the XMM-Newton and Chandra Era [arXiv: astro-ph/0202017]

Shapiro, S. L., Lightman, A. P., \& Eardley, D. M. 1976, ApJ, 204, 187

Schaaf, R., Pietsch, W., Biermann, P. L., et al. 1989, ApJ, 336, 777

Strickland, D. K., \& Stevens, I. R. 2000, MNRAS, 314, 511

Strüder, L., Briel, U., Dennerl, K., et al. 2001, A\&A, 365, L18

Suchkov, A. A., Balsara, D. S., Heckman, T. M., \& Leitherer, C. 1996, ApJ, 430, 511

Sukumar, S., Velusamy, T., \& Klein, U. 1988, MNRAS, 231, 765

Sunyaev, R. A., \& Titarchuk, L. G. 1980, A\&A, 86, 121

Terashima, Y. \& Wilson, A. S. 2004, ApJ, 601, 735

Turner, M. J. L., Abbey, A., Arnaud, M., et al. 2001, A\&A, 365, L27

Turner, T. J., Perola, G. C., Fiore, F., et al. 2000, ApJ, 531, 245

Urry, C. M., \& Padovani, P. 1995, PASP, 107, 803

Veilleux, S. 2001, in Starburst Galaxies: Near and Far, ed. L. Tacconi \& D. Lutz (Heidelberg: Springer-Verlag), 88

Vignati, P., Molendi, S., Matt, G., et al. 1999, A\&A, 349, L57

Zezas, A. L., Fabbiano, G., Rots, A. H., \& Murray, S. S. 2002, ApJ, 577,710 